OPEN ACCESS

Edited by:

Valerie Moyra Pomeroy,

University of East Anglia,

United Kingdom

Reviewed by:

Martin Ebinger

Charité Medical University of

Berlin, Germany

Nicola J. Hancock

University of East Anglia,

United Kingdom

*Correspondence:

Christina Kruuse

ckruuse@dadlnet.dk

Specialty section:

This article was submitted to

Stroke,

a section of the journa

Frontiers in Neurology

Received: 14 February 2019 Accepted: 06 June 2019

Published: 28 June 2019

Citation:

Steen Krawcyk R, Vinther $A$

Petersen NC, Faber J, Iversen HK,

Christensen T, Lambertsen KL,

Rehman S, Klausen TW, Rostrup E

and Kruuse C (2019) Effect of

Home-Based High-Intensity Interval

Training in Patients With Lacunar Stroke: A Randomized Controlled Trial.

Front. Neurol. 10:664.

doi: 10.3389/fneur.2019.00664

\section{Effect of Home-Based High-Intensity Interval Training in Patients With Lacunar Stroke: A Randomized Controlled Trial}

Rikke Steen Krawcyk ${ }^{1,2}$, Anders Vinther ${ }^{1,3}$, Nicolas Caesar Petersen ${ }^{4}$, Jens Faber ${ }^{5}$, Helle K. Iversen ${ }^{6}$, Thomas Christensen ${ }^{7}$, Kate Lykke Lambertsen ${ }^{8,9,10}$, Shazia Rehman ${ }^{11}$, Tobias Wirenfeldt Klausen ${ }^{12}$, Egill Rostrup ${ }^{13}$ and Christina Kruuse ${ }^{2 *}$

${ }^{1}$ Department of Physiotherapy and Occupational Therapy, Herlev Gentofte Hospital, University of Copenhagen, Copenhagen, Denmark, ${ }^{2}$ Neurovascular Research Unit, Department of Neurology, Herlev Gentofte Hospital, University of Copenhagen, Copenhagen, Denmark, ${ }^{3} \mathrm{QD}$-Research Unit, Herlev Gentofte Hospital, University of Copenhagen, Copenhagen, Denmark, ${ }^{4}$ Center for Translational Neuromedicine, University of Copenhagen, Copenhagen, Denmark, ${ }^{5}$ Division of Endocrinology, Department of Internal Medicine, Faculty of Health and Medical Sciences, Herlev Gentofte Hospital, University of Copenhagen, Copenhagen, Denmark, ${ }^{6}$ Department of Neurology, Stroke Center Rigshospitalet, University of Copenhagen, Copenhagen, Denmark, ${ }^{7}$ Department of Neurology, Nordsjællands Hospital, University of Copenhagen, Copenhagen, Denmark, ${ }^{8}$ Department of Neurobiology Research, Institute of Molecular Medicine, University of Southern Denmark, Odense, Denmark, ${ }^{9}$ Department of Neurology, Odense University Hospital, Odense, Denmark, ${ }^{10}$ BRIDGE-Brain Research Interdisciplinary Guided Excellence, Department of Clinical Research, University of Southern Denmark, Odense, Denmark, ${ }^{11}$ Department of Radiology, Herlev Gentofte Hospital, University of Copenhagen, Copenhagen, Denmark, ${ }^{12}$ Department of Haematology, Herlev Gentofte Hospital, University of Copenhagen, Copenhagen, Denmark,

${ }^{13}$ Mental Health Center Glostrup, Copenhagen, Denmark

Background: High-intensity interval training $(H \| I T)$ is superior to moderate-intensity continuous training in improving cardiorespiratory fitness in patients with cardiovascular disease, but is it safe, feasible and effective in patients with stroke? We investigated feasibility and effect of early, home-based HIIT in patients with lacunar stroke combined with usual care vs. usual care, only.

Methods: Patients with minor stroke (severity: 55/58 point on the Scandinavian Stroke Scale) were randomized to HIIT or usual care in a randomized, controlled trial. We measured the following outcomes at baseline and post-intervention: cardiorespiratory fitness monitored as power output from the Graded Cycling Test with Talk Test (GCT-TT; primary outcome), physical activity, fatigue, depression, well-being, stress, cognition, endothelial function, blood pressure, body mass index, and biomarkers.

Results: We included 71 patients (mean age $63.7 \pm 9.2$ ), 49 men, 31 in intervention group. Home-based HIIT was feasible with no reported adverse events in relation to the intervention. No significant change between the groups in GCT-TT power output was detected $(p=0.90)$. The change in time spent on vigorous-intensity activity was $2 \mathrm{~h} /$ week and $0.6 \mathrm{~h} /$ week, intervention and usual care, respectively $(p=0.045)$. There were no significant differences between groups in the remaining secondary outcomes. 
Conclusion: HIIT was feasible and safe in patients with lacunar stroke. Patients can engage early in home-based HIIT when involved in choosing exercise modality and guided by weekly motivational phone calls. Within 3 months, HIIT did, however, not yield effect on cardiorespiratory fitness. We await further evaluation of long-term effects of this intervention on continued regular physical exercise and cardiovascular event.

Clinical Trial Registration: https://clinicaltrials.gov, identifier NCT02731235

Keywords: aerobic exercise, high-intensity interval training, home-based physical activity, secondary stroke prevention, lacunar stroke

\section{INTRODUCTION}

Stroke is one of the leading causes of mortality and disability (1). Approximately $90 \%$ of strokes are attributed to modifiable risk factors, and $75 \%$ of the global stroke burden may be avoided by control of behavioral and metabolic risk factors (2). In stroke survivors, lifestyle modifications combined with preventive medication are recommended to be initiated early, as there is a $3.7-6.7 \%$ risk of recurrence within the first 90 days after stroke onset $(3,4)$. The international multicentre study INTERSTROKE (5) highlighted ten common modifiable risk factors associated with ischemic stroke and identified physical inactivity as one of the most important. Several studies have shown that physical activity has a protective effect against stroke $(6,7)$, as physical activity has the potential to improve cardiorespiratory fitness and reduce blood pressure, lipids, and body weight, thus improving cardiovascular health $(8,9)$. Physical activity and stroke risk factors can evoke changes in inflammatory, endothelial and cardiovascular biomarkers, which may be used to monitor risk factor load, disease progression, or effect of specific interventions (10).

Increasing the physical activity, including increasing the frequency, volume, and intensity of exercise, is associated with health benefits (11) and decreased risk of a future stroke in healthy individuals (12-14). Whether this effect also applies to risk of a recurrent stroke in patients with a manifest cerebrovascular event has yet to be fully established (15).

Following stroke, a low level of physical activity (16) and aerobic exercise (17) is often reported, perhaps due to specific barriers to physical activity after stroke. The most frequently reported barriers are environmental barriers, such as

\footnotetext{
Abbreviations: AI, Augmentation index; ANCOVA, Analysis of covariance; BMI, Body mass index; cLDA, Constrained longitudinal data analysis; CT, Computed tomography scan; GCT-TT, Graded Cycling Test with Talk Test; HIIT, Highintensity interval training; ICAM-1, Intercellular adhesion molecule-1; IL-6, Interleukin-6; IQR, Interquartile range; MDI, Major depression inventory; MET, Metabolic equivalent of task; MFI-20, Multidimensional fatigue inventory; MICE, Moderate-intensity continuous training; MoCA, Montreal cognitive assessment; MRI, Magnetic resonance imaging; PPS, Pressure pain sensitivity; Pro-ADM, Pro-adrenomedullin; Pro-ANP, Pro-atrial natriuretic peptide; RHI, Reactive hyperaemia index; SD, Standard deviation; $\mathrm{SEM}_{95}$, Standard error of measurement with $95 \%$ certainty; SRD, Smallest real difference; TIA, Transient ischemic attack; TNF, Tumor necrosis factor; TOAST, Trial of Org. 10172 in acute stroke treatment; VCAM-1, Vascular cell adhesion molecule-1; VEGF, Vascular endothelial growth factor.
}

challenges in ambulation and transport to the required training facilities, and personal barriers, including lack of motivation and knowledge on how to initiate and maintain an exercise program. Some patients also indicate a fear of recurrent stroke with increased exercise $(18,19)$. A poorer outcome of very early, within 24-h of stroke onset, initiated intensive mobilization has pushed this concern further forward (20).

High-intensity interval training (HIIT) is an exercise modality that could be feasible in patients with stroke due to the low time commitment involved. HIIT has been shown to be a powerful alternative to moderate-intensity, continuous training (MICE) to improve cardiorespiratory fitness in cardiac rehabilitation (21). In addition, improved endothelial function after HIIT compared with MICE has also been reported based on flow-mediated dilation of the brachial artery in patients with cardiovascular disease (22). Based on these studies, we hypothesized that a HIIT programme designed to overcome the typical barriers for physical activity in stroke patients is feasible and could improve cardiorespiratory fitness, participation in physical activity, endothelial function, and quality of life in patients with lacunar stroke. We aimed to investigate the effect of early initiated HIIT for 12 weeks post-stroke in addition to usual care compared with the effects of usual care only.

\section{METHODS}

\section{Study Design}

We designed a randomized controlled trial with a parallelgroup design. Patients were randomized at a $1: 1$ ratio to either intervention or usual care, within 3 weeks of a stroke. Following randomization, patients were followed for 3 months. The reporting of this study adheres to the CONSORT statement (23).

\section{Ethical Approval}

The trial was approved by The Danish Data Protection Agency (ID: HGH-2015-021) and the Research Ethics Committee in the Capital Region of Denmark (Trial Registration number: $\mathrm{H}-15012371)$. Eligible patients provided written informed consent prior to study participation. Furthermore, the study was registered at URL: ClinicalTrials.gov (ID: NCT02731235, registered January 2016). 


\section{Recruitment}

Patients were recruited from January 2016 until January 2018 from the stroke unit at hospitals in the Capital Region of Copenhagen, Denmark; Herlev Gentofte Hospital, Rigshospitalet Glostrup, and Nordsjællands Hospital. All assessments were carried out at the stroke unit at Herlev Gentofte Hospital. During the recruitment period, medical records were screened daily by the study coordinator to identify patients with lacunar stroke. Eligible patients were enrolled consecutively within 1-21 days of stroke onset.

\section{Participants}

Patients 18 years or older diagnosed with a first-time lacunar stroke or a recurrent event of lacunar stroke were enrolled in the study. A lacunar stroke was defined according to the Trial of Org 10172 in Acute Stroke Treatment (TOASTcriteria) (24). This definition included patients with clinical symptoms with a verified relevant brain stem or subcortical hemispheric lesion ( $<2 \mathrm{~cm}$ in diameter in the acute phase) based on computed tomography (CT) scan or magnetic resonance imaging (MRI) scan (25). Additionally, the patients had a severity of neurological symptoms, categorized as "mild" on the Scandinavian Stroke Scale (SSS) (43-58 points) (26). Patients had to speak and read Danish and provide informed consent. We excluded patients with previous large-artery stroke, unstable cardiac condition, atrial fibrillation, pacemaker, uncontrolled hypertension, uncontrolled diabetes, artery stenosis >50\%, symptoms or comorbidities not allowing exercise on a stationary bicycle, dyspnoea caused by heart or pulmonary disease, aphasia, or dementia that interfered with understanding the protocol and physical examinations.

\section{Procedures}

During hospital admission all patients had routine examination for stroke cause and risk factors. These tests included a chest $\mathrm{x}$-ray, 48-h cardiac event monitoring (Novacor, Rueil Malmaison, France), carotid artery imaging (ultrasound), and routine blood tests (full blood count, glucose, electrolytes, lipids, creatinine, etc.). Furthermore, an MRI (sequences: diffusionweighted imaging, the derived apparent diffusion coefficient value, fluid attenuated inversion recovery, and $\mathrm{T} 2 *$-weighted images on a $1.5 \mathrm{~T}$ clinical system using an 8-channel standard head coil) (Achieva, Philips Healthcare, Best, The Netherlands) was acquired to confirm diagnosis, localization, and presence of acute and old ischemic lesions and microbleeds.

All patients complied with medication in accordance with their stroke physicians' recommendation during admission. In addition, all patients were offered usual occupational therapy and physiotherapy during their hospital stay when needed. As part of the usual care procedure, patients were also offered a follow-up visit at the outpatient clinic.

During the trial, we made three study amendments to increase the recruitment rate: (1) we extended the study to also include patients with acute transient ischemic attack (TIA) and concomitant MRI-verified signs of a previous lacunar stroke (5 patients) (previously only patients with acute/subacute stroke were included), (2) we included patients within 21 days after onset of symptoms (previously only included patients within 7 days), and (3) we expanded the number of recruitments sites to three stroke units in the Capital Region of Copenhagen (previously only one stroke unit). Amendment 1 and 2 were made 6 months after trial initiation (June 28th, 2016) and amendment 3 was made 18 months after trial initiation (June 27th, 2017).

\section{Randomization and Blinding}

After completing all assessments at baseline, the patients were randomized into one of two groups: usual care and exercise intervention or usual care only. The randomization procedure was based on equal allocation with randomly varying block size. The block-randomization was computer-generated ( 8 blocks of 10, mixed with 5 blocks of 4 ) and carried out by a research assistant not involved in the study. Sealed opaque envelopes were made by the research assistant, stored, and administrated by health personnel not involved in the study. The outcome assessor, data analysts, and study coordinator were all blinded to the randomization process. Immediately following baseline assessments, the study coordinator collected the next envelope from the health personnel. The consecutively enrolled patient opened the envelope and was allocated to either intervention group or usual care group.

\section{Intervention}

\section{Both Intervention and Usual Care Group}

At baseline, all patients attended a motivational talk with the study coordinator to encourage lifestyle changes, and they were introduced to an exercise catalog with a range of suggestions for modes of aerobic exercise (e.g., brisk walking stair stepping, stationary bicycling, outdoor cycling, running, indoor rowing, high knee exercises, and swimming).

\section{Intervention Group}

In addition to usual care, the intervention group performed home-based HIIT daily $3 \times 3 \mathrm{~min}$ with $2 \mathrm{~min}$ of active recovery, 5 days per week for 12 weeks (27) (Figure S1). HIIT was defined as exercise at $77-93 \%$ of the maximum heart rate, corresponding to $14-16$ on the Borg-rated perceived-exertion scale (28) or "not able to speak comfortably" on the Talk Test (29-31). The Talk Test determined the initial intensity of the intervention, and the patients progressed the work load and the cadence as they improved throughout the exercise program. In each session, the patients were encouraged to reach an exercise intensity at which they were no longer able to speak comfortably. To determine their speaking comfort the intervention group carried a pocketsized, laminated standardized text passage (cue card), which became redundant as they got familiar with the text. They also wore a stop watch to time the exercise intervals. The exercise modality was self-chosen assuming it was performed at high intensity. Patients in the intervention group were provided with a stationary bicycle if required (Kilberry ${ }^{\circledR}$ Magnetic Bike JC-950, Proteus Sports Inc., Linkou Township, Taiwan) for use at home to ensure an easily accessible exercise modality. Before initiating the exercise program, the study coordinator visited each patient at home to introduce the exercise program, including the use of the Talk Test. Furthermore, the exercise sessions were tracked by 
the patients in an exercise diary to encourage adherence to the exercise program and to report any adverse events.

For further motivation and control of completion of workout, the study coordinator contacted the patients during the exercise period, on a weekly basis to ensure compliance, identify equipment malfunction, and to register any adverse events (AE). An AE was defined as any untoward and unintended response during the exercise intervention with serious adverse event (SAE) or without hospital admission, which did not necessarily have a causal relationship to the intervention. We registered (S)AE from start of the intervention, throughout the intervention period weekly until 2 weeks after cessation of the intervention. Events of definite TIA or stroke were considered outcome measures (32).

\section{Usual Care Group}

The usual care group received secondary preventive medication and advice on self-managed lifestyle changes. Furthermore, the usual care group was asked to resume their habitual level of physical activity and to track their physical activity in an exercise diary.

\section{Outcome Measures}

All outcome measures were obtained at baseline and postintervention (3 months after initiation of the intervention) (Figure S2).

\section{Cardiorespiratory Fitness}

The primary outcome, the Graded Cycling Test with Talk Test (GCT-TT), measured sub-maximal cardiorespiratory fitness monitored as power output in Watts (W). GCT-TT was performed on a stationary bicycle (Monark 928E-G3, Vansbro, Sweden) and identified the exercise intensity at which the patient perceived that it was no longer possible to speak comfortably due to excessive breathing. The workload was increased by $15 \mathrm{~W}$ every minute and each minute the patient also recited a standardized text passage (33). When the patient was no longer able to speak comfortably the test terminated. A detailed test protocol has previously been published (34) establishing the feasibility and measurement error for groups [12.9W; standard error of measurement $\left.\left(\mathrm{SEM}_{95}\right)\right]$ and for individuals with lacunar stroke [18.3W; smallest real difference (SRD)] (34). The SRD corresponded to two steps (30W) in the GCT protocol, which represents a change for an individual patient (34).

\section{Post-stroke Fatigue}

Post-stroke fatigue was measured by the Multidimensional Fatigue Inventory (MFI-20), a generic self-report instrument covering five domains of fatigue: general fatigue, physical fatigue, reduced activity, reduced motivation, and mental fatigue (35). We used a cut-off score $\geq 12$ points in the general fatigue-score as a measure of overall fatigue, as proposed in the original development of the scale (35). Higher score indicated higher degree of fatigue (35).

\section{Depression}

Depression severity was assessed by the Major Depression Inventory (MDI). The questionnaire consisted of 12 questions on mood-related symptoms during the previous 2 weeks, with responses provided on a 6-point Likert scale. The total score ranked from 0-50 points. A high score indicated more severe depression (36), a score $>20$ points indicated mild depression, and a score between 15 and 20 points was interpreted as incipient depression (37).

\section{Mental Well-Being}

Mental well-being was measured by the generic World Health Organization-Five Well-being Index (WHO-5) questionnaire (38). WHO-5 included five positive statements with responses given on a 6-point Likert scale, which corresponded to the patient's own experience for the previous 2 weeks. The total score ranked from $0-100$ points and 50 points indicated reduced well-being or long-term stress (39). In prior studies, the Danish population showed an average score of 69 points (38).

\section{Chronic Stress}

Chronic stress was evaluated using an algometer (Ull-Meter ${ }^{\circledR}$, Ull Care, Hellerup, Denmark) with corresponding recording of pain threshold on the sternum, expressed as pressure pain sensitivity (PPS) $(40,41)$. With the patient in a supine position, the most sensitive point on the sternum was identified, and the hand-held algometer was applied to the sternum with gradually increasing intensity until the pain threshold was reached. The algometer automatically transformed the pain threshold into a logarithmic scale of sensitivity from 30-100 PPS units, with a cut-off $\geq 60$ correlating with markers of a stress syndrome (42). The reading on the algometer was blinded to the observer until completion of the assessment $(40,41)$.

\section{Cognition}

We screened for mild cognitive impairments using the Montreal Cognitive Assessment (MoCA), including the following nine cognitive domains: attention, concentration, executive functions, memory, language, visuospatial ability, conceptual thinking, calculations, and orientation. The total score ranked from $0-$ 30 points, where a score $\geq 26$ points was considered normal cognition (43). MoCA was previously shown to be valid and reliable in patients with lacunar stroke or white matter lesions (44).

\section{Endothelial Function and Arterial Stiffness}

Endothelial function was assessed by digital plethysmography to determine the peripheral arterial tonometry using EndoPAT2000 (Itamar Medical Ltd., Caesarea, Israel) and registered as the reactive hyperaemia index $(\mathrm{RHI})$. An $\mathrm{RHI}$ score $>1.67$ was recommended as a cut-off for normal endothelial function in the user manual of the EndoPAT2000 device.

Arterial stiffness was registered as the augmentation index (AI), together with the heart-rate-corrected AI at a heart rate of 75 beats per min (AI@75). Lower scores indicate greater elasticity of the arteries. Endothelial function and arterial stiffness were both calculated using the EndoPAT software package version 3.4.4. All measures were conducted in accordance with conditions described by the manufacturer, and a detailed procedure has previously been described (45).

\section{Blood Pressure}

Baseline blood pressure was measured at each visit after an overnight fast, following $5 \mathrm{~min}$ of rest with the patient in a supine 
position using an automatic blood pressure monitor (Microlife ${ }^{\circledR}$ BP A100/ Microlife ${ }^{\circledR}$ BP A3L Comfort, Widnau, Switzerland). We aimed for a blood pressure $<130 / 90 \mathrm{mmHg}$ as recommended for patients with a recent lacunar stroke or TIA (46).

\section{Biomarkers}

Venous blood was drawn to assess biomarkers associated with cardiovascular and endothelial function, and inflammation. The cardiovascular biomarkers (pro-adrenomedullin [Pro-ADM], pro-atrial natriuretic peptide [Pro-ANP], and copeptin) are biomarkers to regulate the vascular tone and blood pressure $(47,48)$. The inflammatory biomarkers (interleukin-6 [IL-6] and tumor necrosis factor [TNF]), and endothelial biomarkers (intercellular adhesion molecule-1 [ICAM-1], vascular cell adhesion molecule-1 [VCAM-1], vascular endothelial growth factor [VEGF], and E-selectin) supplemented the endothelial function data retrieved from EndoPAT. Blood was centrifuged at $4,000 \mathrm{rpm}$ for $15 \mathrm{~min}$ at $4^{\circ} \mathrm{C}$ within $45 \mathrm{~min}$ after sampling and all samples were stored at $-80^{\circ} \mathrm{C}$ until analysis. Inflammatory biomarkers and endothelial biomarkers were analyzed using commercially available kits from Mesoscale, Rockville, USA (V-PLEX Plus human: IL-6 kit, TNF kit, ICAM-1 kit, VCAM-1 kit, VEGF kit, and E-selectin kit) according to the manufacturer's instructions. Samples were analyzed in duplicates. Prior to measurement, the samples were diluted two-fold in Diluent 41, and MSD Discovery Workbench software was used for analysis (49). The cardiovascular biomarkers were analyzed using commercially available kits and software from BRAHMS GmbH Hennigsdorf, Germany (KRYPTOR compact PLUS human: Pro-ADM kit, Pro-ANP kit, and copeptin kit) according to the manufacturer's instructions. Fasting plasma insulin concentration (50) was determined using a commercially available kit (ELISA kit, DRG Instruments GmbH, Marburg, Germany) according to the manufacturer's instructions.

\section{Body Mass Index}

Body mass index (BMI) was calculated based on height as measured in centimeters and body weight as measured in kilograms (body weight/height ${ }^{2}$ ) using a body composition monitor (OMRON HBF-500-E; Kyoto, Japan). Obesity was defined by a $B M I \geq 30 \mathrm{~kg} / \mathrm{m}^{2}$ and was associated with increased risk of cardiovascular disease and first-time stroke (46).

\section{Physical Activity}

We evaluated physical activity both subjectively using the selfreported questionnaire Physical Activity Scale version 2.1 (PAS2) (51) and objectively using an accelerometer (AX3, Axivity, York, UK). In PAS2, patients reported their average physical activity behavior 2 weeks prior to hospital admission (baseline) and 2 weeks prior to the post-intervention assessment. PAS2 revealed daily time spent on sleep, sitting down at work, standing/walking at work, heavy physical work during working hours, active commuting to/from work, and sedentary behavior, including television watching and reading. Additionally, in PAS2, patients recorded the weekly time spent on light-intensity, moderateintensity, and vigorous-intensity activity during leisure time (51). Each activity corresponded to a specific MET (metabolic equivalent of task) intensity according to the Compendium of Physical Activity (52), which allows physical activity to be calculated as a total 24-h MET score. To estimate the average duration of activity per day, each score from the three leisuretime activities was divided by seven. Total time reported per day spent on the activities was calculated by adding the hours spent on all activities. If the total time reported was below or above $24 \mathrm{~h}$, we added or subtracted time that was not accounted for to the category "light activity," as suggested in a previous study (51).

Objective physical activity was acquired by a wireless threeaxis accelerometer (AX3, Axivity, York, UK) fixed with doublesided adhesive tape (VIP Tape, Skinlock International, Charleroi, Belgium) anteriorly on the right medial thigh with a waterresistant patch (Fixomull ${ }^{\circledR}$ transparent, BSN Medical, Inc., Hamburg, Germany). The AX3 was programmed to record activity patterns for 8 days and 7 nights with a frequency of $25 \mathrm{~Hz}$, using manufacturer's software (Open Movement v.1.0.0.28). After download, data were analyzed with Acti4, a custommade script in MATLAB (version: R2013a), including a previous described algorithm (53) to identify everyday physical activity types, such as walking, running, cycling, walking stairs, standing up, and sitting down.

\section{Other Data Collection}

The following data were collected from the patients or from patient records: age, sex, clinical symptoms at time of hospital admission, age, type and location of the lesion, mobility, family status, occupation, education level, pre-existing diabetes, hypertension and hypercholesterolemia upon hospital admission, and smoking and drinking habits.

\section{Statistical Analyses \\ Sample Size}

Sample size calculation was based on the primary outcome (GCTTT power output). Using a two-tailed 5\% level of significance and a power of $80 \%$, to detect a minimal clinical important average difference of $23 \mathrm{~W}$, a sample size of 84 patients (42 in each group) was needed. With allowance for a dropout rate of $15 \%$, we aimed to enroll 100 patients in total.

No prior studies have established a minimal clinical important difference for the GCT-TT power output. The decision to choose a $23 \mathrm{~W}$ average difference was based on a previous study in cardiac patients (54) reporting that the smallest detectable average change for a group of patients $\left(\mathrm{SEM}_{95}\right)$ was $18.3 \mathrm{~W}$ and the smallest real difference for an individual patient (SRD) was 25.9W (corresponding to two $15 \mathrm{~W}$-steps in the incremental test protocol). An average difference for a group of patients clearly exceeding $23 \mathrm{~W}$ are closer to two incremental steps (30W) than to one $(15 \mathrm{~W})$ incremental step in the test protocol. Thus, it was our best estimate of a minimal clinical important average difference between groups.

\section{Analysis}

We analyzed complete outcome data according to the group the patients were randomized to, regardless of patient compliance. All available data for each patient were included in the analysis. Missing data were not imputed. Data on demographics and 
baseline characteristics were compared between the groups using independent $t$-test for comparison of means and Fisher's exact test for comparison of categorical variables.

To evaluate outcome changes between the groups for both the primary outcome and for secondary outcomes, we used analysis of covariance (ANCOVA) for continuous variables, the Mann-Whitney test for ordinal variables (estimating the difference at the post-intervention assessment), and Fisher's exact test for categorical variables. For evaluation of differences within each treatment group for both primary and secondary outcomes, we used a paired $t$-test for continuous variables and a Wilcoxon signed rank test for ordinal variables. The differences, both between groups and within groups, were calculated and given as mean difference with $95 \%$ confidence intervals (CI). Before analysis, all variables were tested for normal distribution using histograms and QQ-plots, and if necessary, the data were logarithmically transformed. All data were analyzed with a two-tailed test and with a statistical significance level set at $p<0.05$. Data were analyzed using Microsoft Excel 2010 (Microsoft Corporation, Redmond, WA, USA) and IBM SPSS Statistics 22 (Armonk, NY, USA). Statistical planning and analysis were conducted in cooperation with one of the authors, a biostatistician (TWK).

\section{RESULTS}

No significant differences were found between the groups in terms of demographics or baseline characteristics (Table 1), and no adverse events related to the intervention were recorded during the study.

\section{Study Recruitment}

We screened records on all patients admitted to the stroke unit ( $n=3,098)$ daily and excluded 2,969 patients who did not have a diagnosis of lacunar stroke. A total of 58 patients with lacunar stroke declined participation due to the following reasons: reduced mental surplus (the patients were too tired or excused themselves with no energy, as seen with mental fatigue) $(n=21)$, no reason given $(n=25)$, pain $(n=8)$, and work obligations $(n=4)$. Of the declining patients, 31 were men (mean age $70 \pm 9$ years) and 27 women (mean age $69 \pm 11$ years). In total, 71 patients with lacunar stroke were included with a mean symptom severity score (SSS score) on admission of $55 \pm 5$ points. The patients were recruited 1-17 (mean $6 \pm 4$ ) days after stroke onset and the first assessment visit was performed $12 \pm 7$ days after hospital admission.

Between March 2016 and April 2018, 63 patients attended the post-intervention assessment. Eight patients (11\%), four in each group, were lost to the post-intervention assessment. The cause of drop-out in the intervention group were: one patient had adverse event unrelated to the study intervention (a lumbar herniated disc allegedly induced by heavy lifting during a house renovation project), one patient had work obligations, and two patients reported reduced mental surplus. In the usual care group, the cause of drop-out were: one patient had chronic pain in her knee from osteoarthritis and was awaiting surgery, one patient complained of reduced mental surplus, and the remaining gave
TABLE 1 | Patient characteristics at baseline.

\begin{tabular}{|c|c|c|}
\hline Variable & $\begin{array}{l}\text { Intervention } \\
\quad(n=31)\end{array}$ & $\begin{array}{l}\text { Usual care } \\
\qquad(n=32)\end{array}$ \\
\hline Men, $n$ (\%) & $23(74)$ & $26(81)$ \\
\hline Age, years (mean $\pm S D$ ) & $63.7 \pm 8.9$ & $63.7 \pm 9.2$ \\
\hline \multicolumn{3}{|l|}{ Mobility } \\
\hline Without walking aids, $n$ (\%) & $28(90)$ & $30(94)$ \\
\hline Pre-stroke use of walking aids, $n$ (\%) & 0 & 1 (3) \\
\hline Scandinavian stroke scale, points (mean \pm SD) & $54.6 \pm 5.8$ & $55.3 \pm 4.4$ \\
\hline \multicolumn{3}{|l|}{ Family status } \\
\hline Cohabitants, $n(\%)$ & $22(71)$ & $23(72)$ \\
\hline Living alone, $n(\%)$ & $9(29)$ & $9(28)$ \\
\hline \multicolumn{3}{|l|}{ Education } \\
\hline Primary education, $n$ (\%) & 1 (3) & $3(9)$ \\
\hline Apprenticeship, $n(\%)$ & $9(29)$ & $9(28)$ \\
\hline Upper secondary education/high school, $n$ (\%) & $1(3)$ & $1(3)$ \\
\hline Short-cycle tertiary education, $n(\%)$ & $6(19)$ & $2(6)$ \\
\hline Bachelor or equivalent, $n(\%)$ & $9(29)$ & $5(16)$ \\
\hline Masters, equivalent or higher, $n(\%)$ & $5(16)$ & $12(38)$ \\
\hline \multicolumn{3}{|l|}{ Lesion, age } \\
\hline First-time lacunar stroke, $n(\%)$ & $18(58)$ & $17(53)$ \\
\hline Recurrent lacunar stroke, $n(\%)$ & $3(10)$ & $2(6)$ \\
\hline $\begin{array}{l}\text { Only older lacunar infarct verified on MRI, } \\
\text { combined with clinical symptoms } \\
\text { corresponding with a TIA, } n(\%)\end{array}$ & $1(3)$ & $4(13)$ \\
\hline $\begin{array}{l}\text { First-time lacunar stroke but also sequela } \\
\text { lacunar stroke verified on MRI, } n(\%)\end{array}$ & $9(29)$ & $9(28)$ \\
\hline Acute/subacute infarct, $n(\%)$ & $30(97)$ & $28(88)$ \\
\hline Previous clinical symptoms, $n$ (\%) & $8(26)$ & $4(13)$ \\
\hline Thrombolysis, $n$ (\%) & $3(10)$ & $3(9)$ \\
\hline \multicolumn{3}{|l|}{ Hemispheric localization of lesion } \\
\hline Right hemisphere, $n(\%)$ & $19(61)$ & $18(56)$ \\
\hline Left hemisphere, $n(\%)$ & $12(39)$ & $12(38)$ \\
\hline Bilateral, $n(\%)$ & 0 & $2(6)$ \\
\hline \multicolumn{3}{|l|}{ Lesion localization } \\
\hline Thalamus & 9 & 5 \\
\hline Basal ganglia & 17 & 19 \\
\hline Frontal lobe & 2 & 2 \\
\hline Pons & 1 & 4 \\
\hline Medulla oblongata & 1 & 1 \\
\hline Corpus callosum & 0 & 1 \\
\hline Motor cortex & 1 & 0 \\
\hline \multicolumn{3}{|l|}{ Clinical symptoms on admission } \\
\hline Paresis/dexterity of extremities, $n(\%)$ & $23(74)$ & $18(56)$ \\
\hline Sensory impairments of the extremities, $n(\%)$ & $11(35)$ & $12(38)$ \\
\hline Facial palsy, $n(\%)$ & $7(23)$ & $11(34)$ \\
\hline Dysarthria, $n(\%)$ & $6(19)$ & $11(34)$ \\
\hline Vertigo, $n(\%)$ & $5(16)$ & $8(25)$ \\
\hline Visual problems, $n(\%)$ & $5(16)$ & $2(6)$ \\
\hline \multicolumn{3}{|l|}{ Cardiovascular risk factors } \\
\hline Hypertension at hospitalization, $n(\%)$ & $26(84)$ & $25(78)$ \\
\hline Hypertension previously known, $n(\%)$ & $17(55)$ & $14(44)$ \\
\hline Pre-existing diabetes, $n(\%)$ & $3(10)$ & 2 (6) \\
\hline $\mathrm{BMI}, \mathrm{kg} / \mathrm{m}^{2}($ mean $\pm \mathrm{SD})$ & $28 \pm 5$ & $26 \pm 4$ \\
\hline
\end{tabular}


TABLE 1 | Continued

\begin{tabular}{|c|c|c|}
\hline Variable & $\begin{array}{l}\text { Intervention } \\
(n=31)\end{array}$ & $\begin{array}{c}\text { Usual care } \\
\quad(n=32)\end{array}$ \\
\hline \multicolumn{3}{|l|}{ Smoking } \\
\hline Current smokers, $n(\%)$ & $6(19)$ & $6(19)$ \\
\hline Previous smokers, $n(\%)$ & $14(45)$ & $16(50)$ \\
\hline Non-smokers, $n(\%)$ & $11(36)$ & $10(31)$ \\
\hline \multicolumn{3}{|l|}{ Alcohol consumption ${ }^{\star \star}$} \\
\hline$<$ Health authorities' recommendations, $n(\%)$ & $18(58)$ & $21(66)$ \\
\hline$>$ Health authorities' recommendations, $n$ (\%) & $13(42)$ & $11(34)$ \\
\hline \multicolumn{3}{|l|}{ Lipids } \\
\hline Total cholesterol, mmol/L (mean \pm SD) & $5.6 \pm 1.3$ & $5.5 \pm 1.4$ \\
\hline $\mathrm{LDL}, \mathrm{mmol} / \mathrm{L}($ mean $\pm \mathrm{SD})$ & $3.3 \pm 1.3$ & $3.1 \pm 1.0^{*}$ \\
\hline $\mathrm{HDL}, \mathrm{mmol} / \mathrm{L}($ mean $\pm \mathrm{SD})$ & $1.4 \pm 0.5$ & $1.4 \pm 0.4$ \\
\hline Triglycerides, mmol/L (mean \pm SD) & $2.0 \pm 1.1$ & $1.7 \pm 0.8^{*}$ \\
\hline \multicolumn{3}{|l|}{ Blood pressure } \\
\hline Systolic pressure, $\mathrm{mmHg}$ (mean $\pm \mathrm{SD}$ ) & $149 \pm 22$ & $147 \pm 21$ \\
\hline Diastolic pressure, $\mathrm{mmHg}$ (mean $\pm \mathrm{SD}$ ) & $85 \pm 10$ & $89 \pm 11$ \\
\hline
\end{tabular}

No significant difference was detected between the groups.

${ }^{* *}$ The Danish Health authority recommends $<7$ units per week for women ( 1 unit equals 1 glass of wine) and $<14$ units per week for men (55).

$* n=30$.

no cause (Figure 1). The patients lost to the post-intervention assessment had a mean age of $63 \pm 11$ years, five were women, and six lived alone. Of the 63 patients included in the analysis, five patients $(8 \%)$ were readmitted to the hospital within the first 3 months of lacunar stroke. All readmissions were identified as serious adverse events, however unrelated to the intervention (one patient from the intervention group and four from the usual care group). Two patients were identified to have a clinical diagnosis of a new TIA, one patient was admitted for chest pain, and two patients for dizziness and malaise.

The study was terminated as planned, but due to low recruitment, we did not fulfill the intended inclusion number, and extension of inclusion was not possible due to time and finances.

\section{Primary Outcome}

In total, 58 patients were analyzed with the GCT-TT at the post-intervention assessment. Three patients from the usual care group did not complete the GCT-TT, one had fatigue of the paretic leg at baseline, prior to any intervention, one had longstanding knee osteoarthritis and refused to perform the GCT-TT at the post-intervention assessment for the fear of worsening the pain, and one patient did not complete due to technical problems with the stationary bicycle. Two patients from the intervention group did not complete the GCT-TT: one because of nausea, dizziness, and stomach ache on the day of the post-intervention assessment considered not to be related to the intervention. The fourth patient experienced acute lower back pain at the day of post-intervention assessment and was not able to sit on the stationary bicycle (Figure 1).

There was no significant difference over time between groups in GCT-TT power output ( $p=0.90$; Table 2$)$. However, 17 of 29 patients in the intervention group improved their GCT-TT power output from baseline to post-intervention assessment, and 11 of 29 patients in the usual care group improved their GCT-TT power output from baseline to the post-intervention assessment $(p>0.05$; data not shown). Eight patients in each group improved $\geq 30 \mathrm{~W}$ in GCTTT power output, and this change corresponded to two steps in the GCT-TT protocol, equivalent to a clinically relevant change.

\section{Secondary Outcomes Physical Activity}

At baseline, 21 patients in the intervention group and 18 patients in the usual care group reported that they did not spend time on vigorous-intensity activity. We found a significant behavioral change in time spent on vigorous-intensity activity (PAS2) in the intervention group compared with the usual care group from baseline to the post-intervention assessment ( $p=0.045$; Table 2).

In the intervention group, 18 patients increased (58\%), 6 patients decreased (19\%), and 7 patients (23\%) did not change their participation in vigorous-intensity activity. In the usual care group 9 patients $(28 \%)$ increased, 7 patients decreased $(22 \%)$, and 16 patients $(50 \%)$ did not change their participation in vigorous-intensity activity (Figure 2). A higher proportion of patients in the intervention group increased their participation in vigorous-intensity activity from baseline to the post-intervention assessment $(p=0.01)$.

In the intervention group, 15 of 21 patients (71\%) and 5 of 18 patients in the usual care group (28\%) went from, not participating in vigorous-intensity activity at baseline to participate in any vigorous-intensity activity at the postintervention assessment. Furthermore, we found that more patients both from the intervention group and the usual care group performed more physical exercise at the post-intervention assessment compared to baseline. The most substantial positive change was seen for the intervention group regarding their increase in vigorous-intensity activity (Figure 3).

\section{General Well-Being and Cardiovascular Function}

No change was detected between groups in the level of poststroke fatigue, chronic stress, depression, mental well-being, cognition, blood pressure or endothelial function (Table 2).

\section{Biomarkers}

Statistically significant reductions in lipids were observed within both groups ( $p=0.00, p=0.00$, respectively) (Table 3 ). Fasting insulin was reduced in both groups over time, however the decrease in the usual care group was marginally reduced compared to the intervention group $(p=0.048)$. A significant reduction in ICAM-1 in the usual care group compared to the intervention group ( $p=0.006)$ and VCAM-1 was slightly increased in the intervention group compared to the usual care group ( $p=0.024$ ) (Table 3 ). The changes were, however, small with no consistent effect on specific functions (inflammation, endothelium and cardiovascular).

\section{Exercise Adherence}

According to the exercise diaries, patients in the intervention group reported that they, on average, performed the exercise 


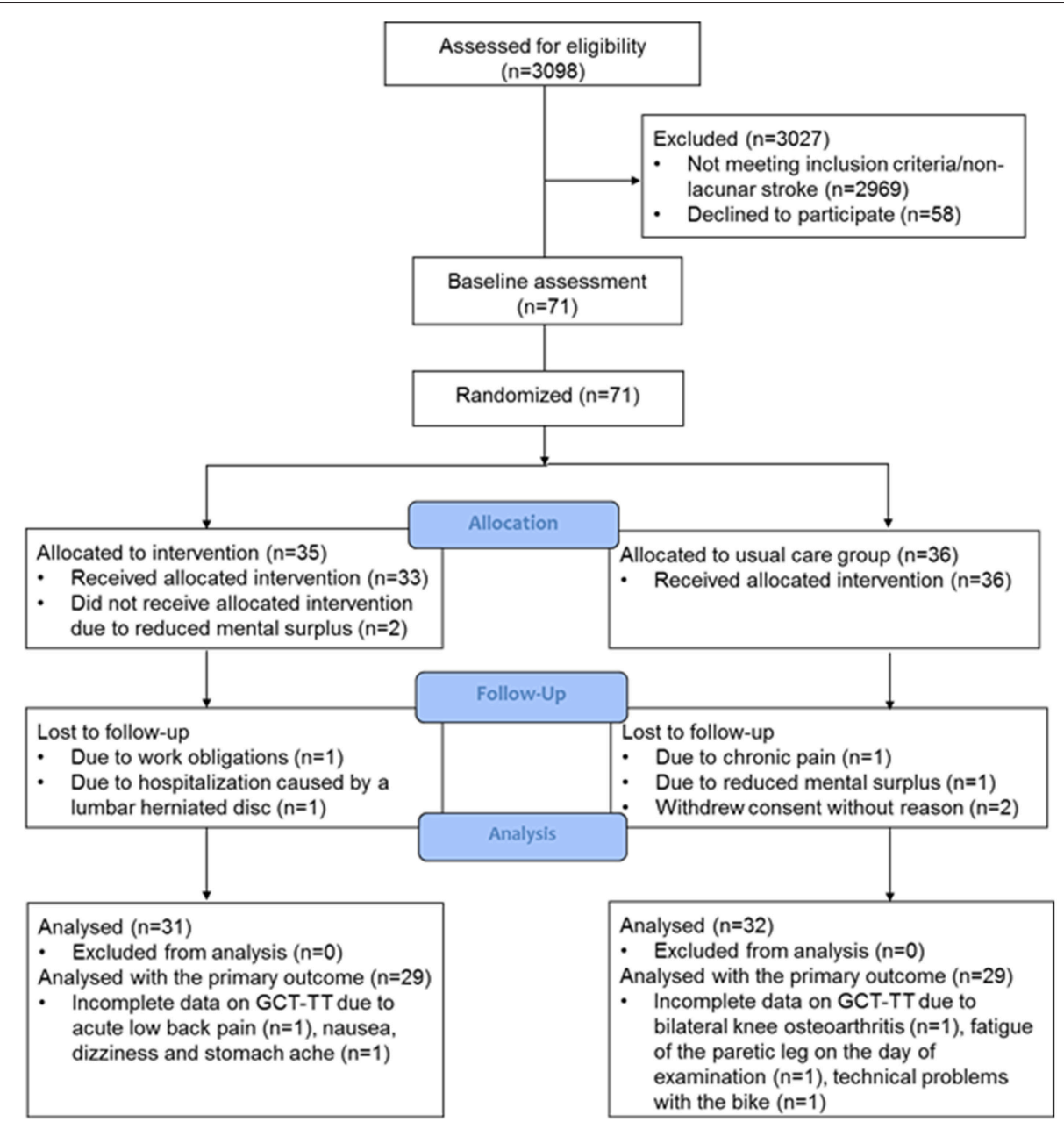

FIGURE 1 | Flow diagram of the randomized controlled trial to investigate effect of home-based HIIT in patients with lacunar stroke.

program 56 of 60 days (93\%). For reference, 60 days of exercise corresponds to 5 days per week for 12 weeks. In the intervention group, 10 of 31 patients (32\%) performed the exercise program more than 5 days per week ( $>100 \%$ adherence), while 24 patients (77\%) in the intervention group performed the exercise program $\geq 4$ days per week ( $\geq 80 \%$ adherence).

A total of 23 patients (74\%) in the intervention group chose to complete the aerobic exercise sessions on the provided stationary bicycle, whereas the remaining 8 patients performed brisk walking (1 patient), stair stepping in combination with outdoor cycling on different days (2 patients), running (2 patients), brisk walking combined with outdoor cycling on different days (1 patient), brisk walking combined with rehabilitation twice a week in the community (1 patient), and indoor rowing (1 patient).

According to the patients' exercise diaries, in which they recorded the number of days with any kind of activity during the 12-week study (84 days), the intervention group reported that they were physically active $64 \pm 12$ days (5.4 days per week), whereas the usual care group reported physical activity on $51 \pm 24$ days (4.2 days per week). Physical activity, measured objectively with accelerometers, did not show a significant difference within or between the groups from baseline to the post-intervention assessment (Table 2).

\section{DISCUSSION}

The main findings of this randomized controlled trial on homebased HIIT were that an early initiated HIIT exercise program was feasible and safe in patients who have had a minor stroke (lacunar stroke), and that a significant proportion of patients in the intervention group markedly increased their time spent on vigorous-intensity activity compared to patients in the usual care group. This reported increase in physical activity, however, was not translated into an improvement in GCT-TT power output. HIIT did not significantly improve the general wellbeing (depression, chronic stress, post-stroke fatigue, cognition, and quality of life), cardiovascular function (blood pressure and endothelial function). For biomarker outcomes (cardiovascular, inflammatory, and endothelial) the results were ambiguous with no obvious beneficial effect of HIIT on the endothelial response. 
TABLE 2 | Results for outcomes measured at baseline and at the post-intervention assessment for both groups.

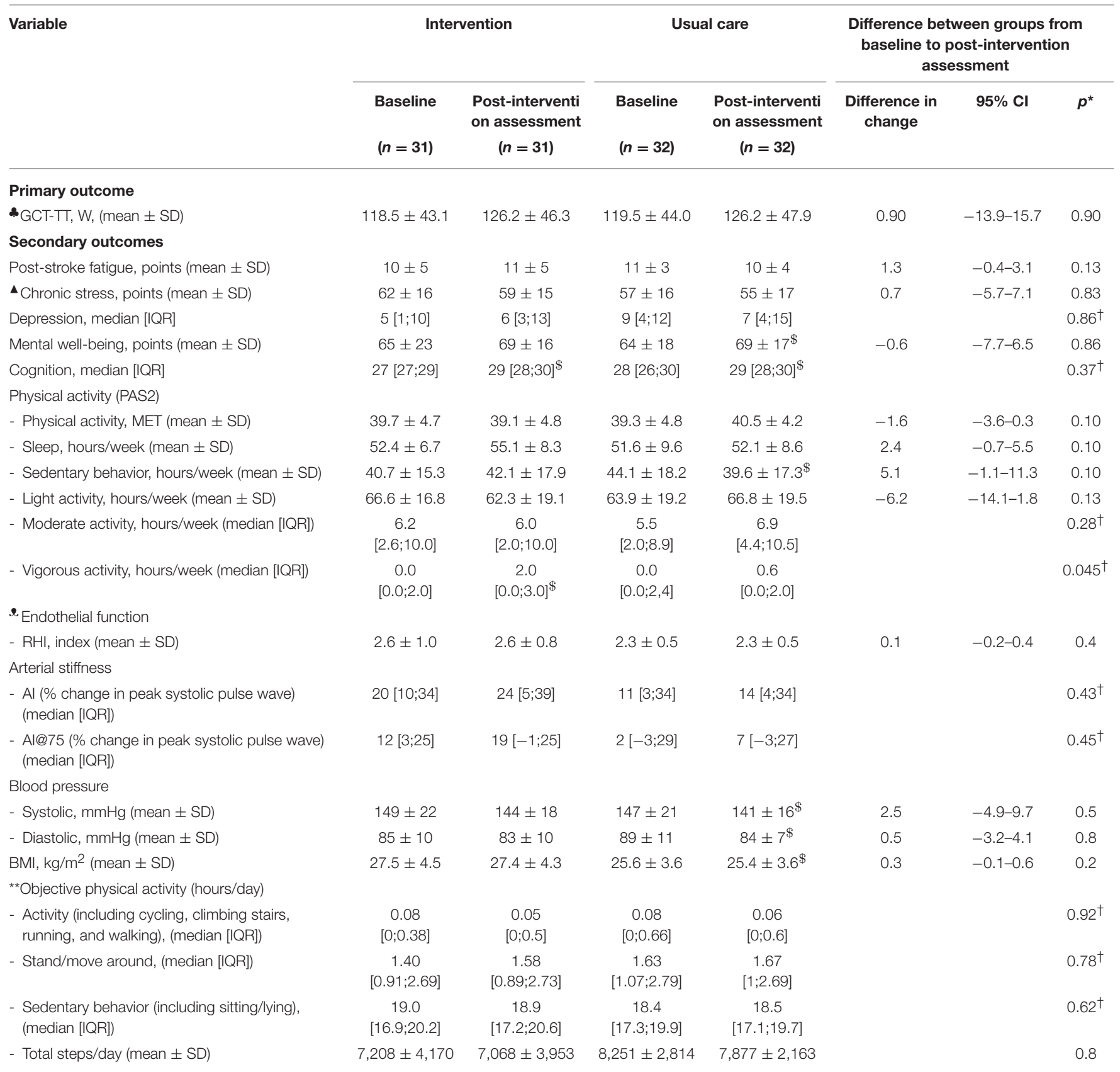

Difference in change is only provided when a mean difference could be calculated.

IQR, interquartile range, ${ }^{*}$ calculated by ANCOVA, ${ }^{\dagger}$ calculated by Mann-Whitney test (between the groups at the post-intervention assessment), *29 patients in each group, ${ }^{\$}$ data from within-group analysis are significant, ${ }^{* *} 26$ patients in each group, ${ }^{\mathfrak{2}} 30$ patients in each group, $\mathbf{} 31$ patients in the usual care group.

Only a few studies have investigated the effect of HIIT in stroke patients (56), and only three studies were randomized controlled trials (57-59). In the present study, we have investigated HIIT as a home-based intervention with distant supervision for patients with lacunar stroke. HIIT is more often performed as individual treadmill training after stroke to improve mobility, gait speed, and gait stride (56). Only one randomized controlled trial reported a superior effect of HIIT vs. MICE to improve cardiorespiratory fitness in patients with stroke (57). More studies that evaluate the effects of the various available exercise methods are needed to confirm these findings. The current study highlights the need for additional research to investigate the effect of HIT on cardiorespiratory fitness in stroke patients.

The exercise intensity was not monitored during the homebased training and too low exercise intensity may explain 
20

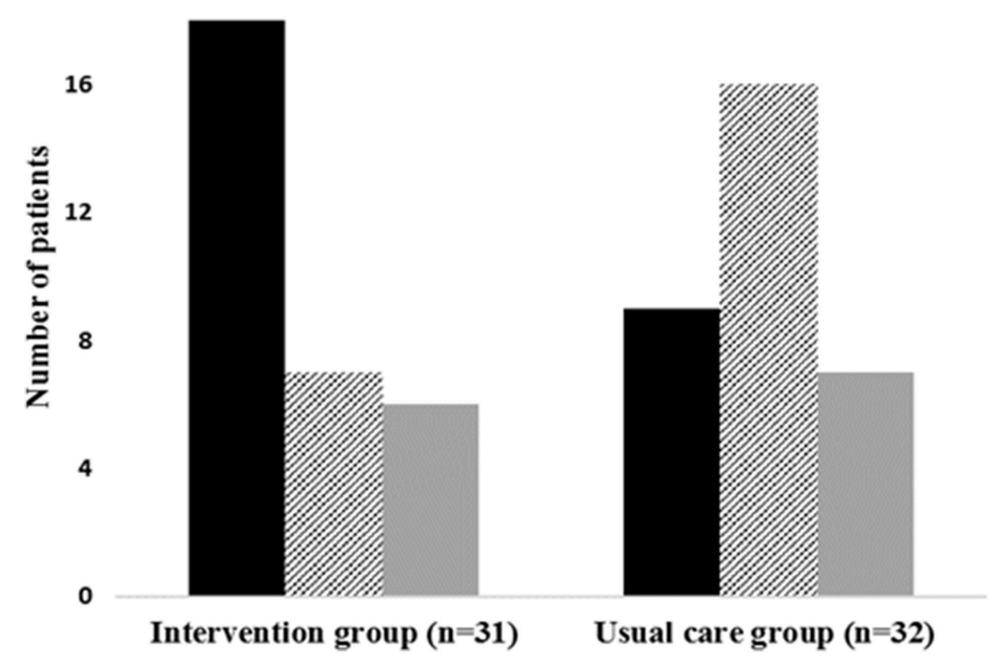

- Increased

"Unchanged

- Reduced

FIGURE 2 | Change in vigorous-intensity activity from baseline to the post-intervention assessment in both groups. The figure shows the number of patients in each group who either increased, did not change or reduced their time spent on vigorous-intensity activity from baseline to post-intervention assessment.

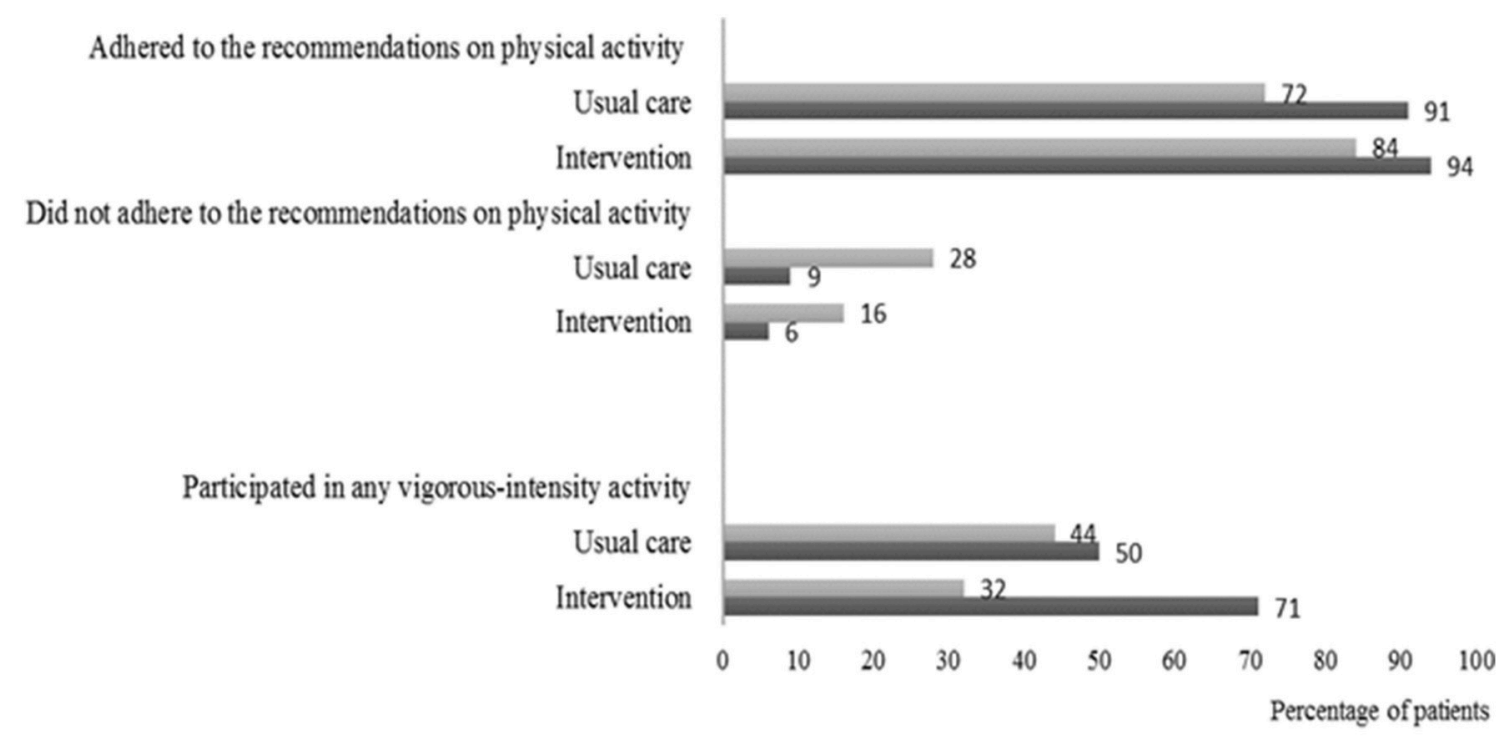

\#aseline $(\mathrm{n}=63) \quad$ = Post-intervention assessment $(\mathrm{n}=63)$

FIGURE 3 | Self-reported adherence to physical activity. Percentages of patients in the intervention group and in the usual care group who adhered or did not adhere to the international recommendations on physical activity at baseline and at the post-intervention assessment. The patients were considered adherent if they performed vigorous-intensity activity ( $\geq 75$ min per week), moderate-intensity activity ( $\geq 150$ min per week), or an equivalent combination. The figure also shows the percentage of patients in the intervention group and in the usual care group who participated in any vigorous-intensity activity at baseline and at the post-intervention assessment.

part of the missing cardiovascular effects. We chose not to measure the exercise intensity with heart rate monitors based on clinical observations in a small test of feasibility which showed that the patients with lacunar stroke were not able to comply with the available monitoring equipment. Their familiarity with, and ability to use smartphones was not consistent, and some patients developed a skin rash following use of electrocardiography electrodes for more than 3-5 days. Furthermore, the commercially available heart rate monitors at the initiation of the study did not have the required memory capacity or battery life for weeklong monitoring. 
TABLE 3 | The results for biomarkers measured at baseline and at the post-intervention assessment for both groups.

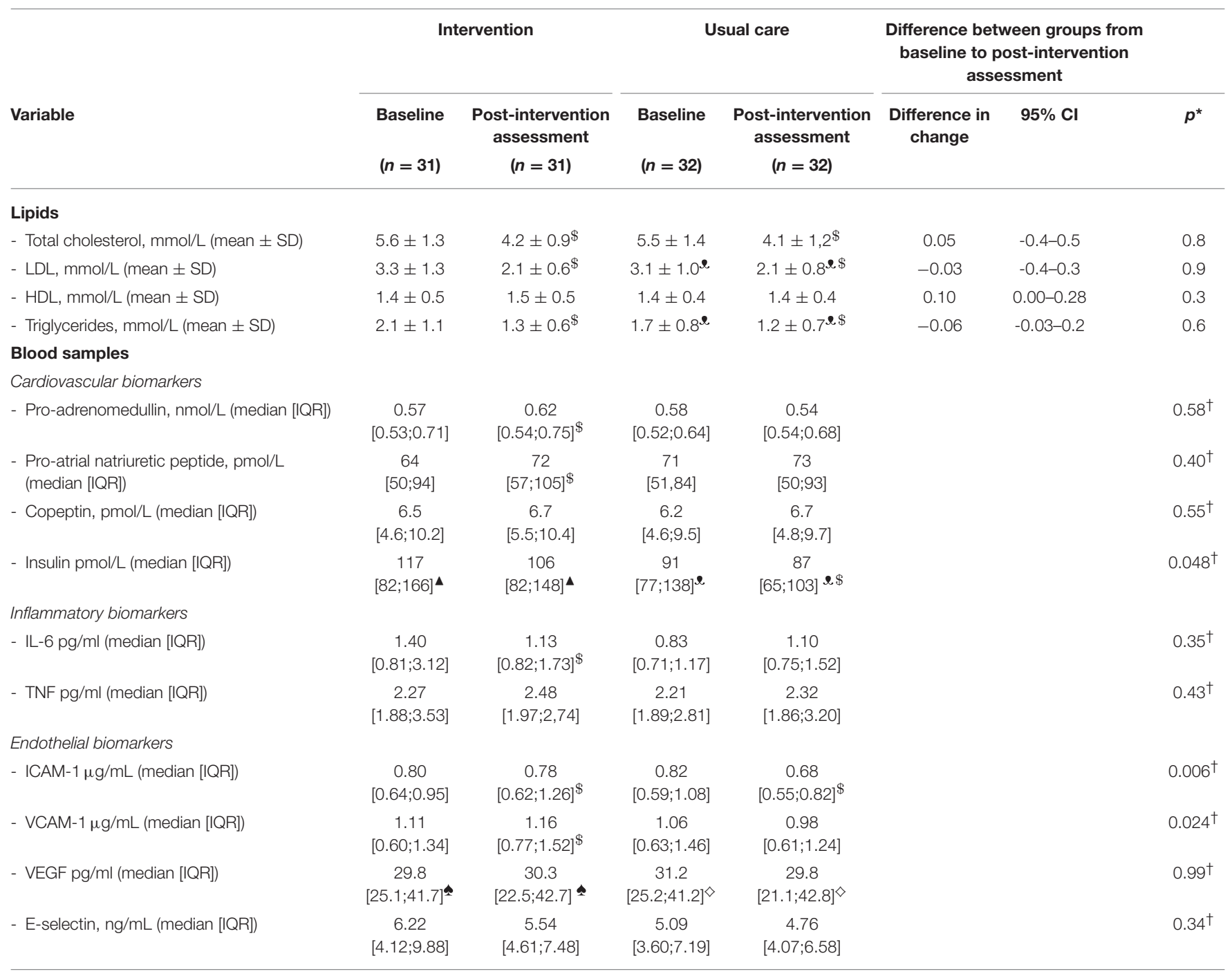

Difference in change is only provided when a mean difference could be calculated.

IQR, interquartile range, ${ }^{*}$ calculated by ANCOVA, ${ }^{\dagger}$ calculated by Mann-Whitney test (between the groups at the post-intervention assessment), ${ }^{\$}$ data from within-group analysis are significant, $\diamond_{n}=25, \boldsymbol{\bullet}_{n}=28, \boldsymbol{\bullet}_{n=29}, \boldsymbol{\imath}_{n}=30$.

Another cause of discrepancy between reported activity and lack of cardiovascular effects may be the relatively short exercise duration ( $15 \mathrm{~min}$ of exercise 5 days a week). When planning the study, our hypothesis was that patients with lacunar stroke were likely to be unfamiliar with physical activity and feasibility of the intervention was a main priority. There is no general consensus on the ideal HIIT protocol for motor recovery and improved cardiorespiratory fitness after stroke (60). The most frequently used HIIT protocol in patients with cardiovascular disease targeting endothelial function, was $4 \times 4 \mathrm{~min}$, three times per week (22). Additionally, adherence to the international recommendations on physical activity for health, require $75 \mathrm{~min}$ of weekly vigorous-intensity activity (61), thus we chose $15 \mathrm{~min} 5$ times a week.

Furthermore, the current home-based HIIT intervention was designed to be easy for patients to perform at home, easy to translate into clinical practice, and at low-cost in an effort to reduce the known barriers for physical activity in stroke survivors $(18,19)$.

All patients, also those in the usual care group, were aware of being enrolled in an exercise study with regular assessments, and all patients were informed on the importance of physical activity following stroke. These features of the study may have encouraged all patients to be physically active. In addition, the supervision of all patients by a physiotherapist during the baseline assessment of the GCT-TT may have decreased the fear of doing aerobic exercise following stroke also in the usual care group. Despite encouragements and a subtle increase in physical activity, a change exceeding the previously established $\mathrm{SEM}_{95}$ of $12.9 \mathrm{~W}$ (34) was not observed either within the groups or between the groups in GCT-TT power output.

Patients with an ischemic stroke or a transient ischemic attack (TIA) have an increased risk of recurrent stroke and cardiovascular events (62). Consequently, medical intervention 
or change in lifestyle factors are often suggested. Secondary prevention using medication is effective in preventing recurrent stroke (46). However, the evidence exploring the effect of lifestyle changes on recurrent stroke is not solid (46). Two recent reviews explored lifestyle interventions, including cardiovascular exercise to prevent cardiovascular events after stroke and TIA (63). They found no effect on cardiovascular events, but a significant reduction in systolic blood pressure, fasting insulin and fasting glucose, and an increase in high-density lipoprotein cholesterol $(63,64)$. However, our study was not able to confirm similar significant findings on cardiovascular function.

A priori we were curious to see if we could detect an improvement in general well-being (depression, chronic stress, post-stroke fatigue, cognitive function, and mental well-being) in favor of the intervention group. We did, however not detect a significant improvement between the groups within the first 3 months of stroke. The few previous studies reporting on the efficacy of exercise (aerobic exercise and resistance training) on depression and well-being showed inconsistent results (65), though a trend toward exercise having a positive impact on cognitive function has been suggested (65). There is not yet sufficient literature available on the efficacy of nonpharmacological interventions to prevent or treat post-stroke fatigue (66), likewise there is a lack of knowledge on the effect of exercise on stress, none of these outcomes were affected in this study, however, the baseline values were surprisingly good leaving little room for possible improvement. Of note, the sample size was small, and the effects of HIT may become more consistent and significant in a larger sample size.

Studies have showed that HIIT had a protective effect on endothelial function in patients with cardiovascular disease (22). Also, aerobic exercise reduces the blood pressure in patients with stroke or TIA (67). Consequently, we expected an improvement on endothelial function in favor of the intervention group, but such was not detected within the 3 months of exercise. It may be related to a potentially low exercise intensity and to a short daily exercise duration but may also be caused by a low incidence of or too well-managed co-morbidities to decrease endothelial function such as diabetes or hypertension in our patient population.

Based on the literature investigating patients with cardiovascular disease, we primarily expected a decrease in biomarkers following physical exercise. Inflammatory biomarkers (IL-6 and TNF) were shown to decrease in patients with coronary heart disease $(10,68)$. Endothelial biomarkers, ICAM-1, VCAM-1 $(68,69)$, VEGF $(70,71)$ were all shown to decrease in patients with cardiovascular risk factors, whereas there was insufficient evidence on the efficacy of exercise on E-selectin. Cardiovascular biomarkers (pro-ADM, pro-ANP) were shown to decrease in patients with heart failure $(72,73)$, and limited literature is available on the efficacy of exercise on Copeptin. We found only minor changes in the assessed biomarkers without clinical relevance between the groups. When comparing the baseline values from the included stroke patients to values from healthy individuals (manufacturer's normative values), the levels of inflammatory, cardiovascular biomarkers and ICAM-1 were generally higher in the stroke patients. This difference may reflect the underlying cardiovascular risk factors in our patient sample.

During the study period, we found an $8 \%$ rate of hospital readmittance, of which two patients (3\%) had a confirmed clinical diagnosis of TIA. In comparison, previous studies have identified a risk of $3.7-6.7 \%$ of stroke recurrence within the first 90 days after stroke onset $(3,4)$. This is interesting, and it could be speculated that patients with minor stroke may be less predisposed to stroke recurrence compared to patients with larger stroke severity. Prospective studies with large sample sizes are, however, needed to further elucidate this.

By providing the patients with a stationary bicycle, contacting them on a weekly basis and monitoring them at baseline, we succeeded in engaging patients with lacunar stroke into being more physically active, including performing vigorous-intensity activity. These findings are very encouraging compared to the results of a large international multicentre study (ExStroke), which found no effect of repeated encouragement and verbal instructions on physical activity in patients with ischemic stroke (74). In addition, the Look AHEAD study (75) did not show an effect on cardiovascular events when aiming for lifestyle changes. The study investigated weight reduction by eating fewer calories combined with increased physical activity (unsupervised homebased exercise) by using repeated encouragement (individual sessions and group meeting) in patients with type 2 diabetes. In Look AHEAD, half of the study population dropped out or had no change in physical activity. These results highlight the difficulties of encouraging individuals to make lifestyle changes and the challenges in maintaining them.

\section{STRENGTHS AND LIMITATIONS}

The tailormade HIIT intervention performed in the patients' home environment was considered a strength of this study in contrast to supervised group sessions performed on specific days and hours at a designated training facility, which would likely interfere with non-flexible working hours and transportation if the patients are not allowed to drive after stroke. The choice of no supervision and monitoring of the daily exercise intensity was, however, a limitation of the present intervention. Also, only 8 days of physical activity were monitored using accelerometers rather than the entire intervention period. We supplemented the accelerometers with use of self-reported diaries, which may be subject to recall bias and subconsciously encourage the patients to report better performance to meet own expectations or those of the researcher. In this study we failed to recruit the power-estimated predetermined number of patients due to a low recruitment rate. Since no effect of exercise was detected ( $p=$ 0.90 ), it is highly unlikely that a statistically significant difference would have been reached by inclusion of 15 more patients in each group. Recruitment bias may have occurred since patients, who were already interested in physical exercise were probably more likely to participate in a study investigating an exercise intervention. Another point to raise is the sex distribution of the sample as the majority of the included patients were male (78\%), which could influence the generalisability of the findings. 
However, the present sample reflects the general population of patients with stroke quite well-i.e., higher incidence of stroke in younger men compared to aged-matched women (76). Also, the included patients have had a minor stroke (lacunar stroke) which limits the generalisability of the results to other categories of stroke patients.

\section{CONCLUSION}

The home-based HIIT protocol in the present study was safe and well-received by the patients. The compliance was good leading to increased vigorous-intensity physical activity and training on a daily basis. This increase in physical activity was however not translated into an effect in cardiorespiratory fitness, general well-being, or improvement in biomarkers within the first 3 months of training. This may be caused by a small sample size, insufficient intensity of exercise or a bias in selection of patient who were already physically active on enrolment. Further studies investigating how this exercise approach can be optimized with special emphasis on monitoring of exercise intensity, and on finding the ideal training volume, are warranted to develop an effective HIIT protocol in patients with lacunar stroke.

\section{DATA AVAILABILITY}

The datasets for this manuscript are not publicly available because: according to The Danish Data Protection Agency, a general sharing of patient data is not allowed. Requests to access the datasets should be directed to Christina Kruuse, ckruuse@dadlnet.dk.

\section{ETHICS STATEMENT}

This study was carried out in accordance with the recommendations of the CONSORT statement, with written informed consent from all subjects. All subjects gave written informed consent in accordance with the Declaration of Helsinki. The protocol was approved by the research ethics committee in the Capital Region of Denmark.

\section{AUTHOR CONTRIBUTIONS}

$\mathrm{CK}$ and $\mathrm{AV}$ contributed substantial to the study conception and design, supervised and facilitated all work performed at the stroke unit, Herlev Gentofte hospital and provided key intellectual content to the manuscript. CK and RS obtained

\section{REFERENCES}

1. Benjamin EJ, Blaha MJ, Chiuve SE, Cushman M, Das SR, Deo R, et al. Heart disease and stroke statistics-2017 update: a report from the American Heart Association. Circulation. (2017) 135:e146-603. doi: 10.1161/CIR.0000000000000485

2. Feigin VL, Roth GA, Naghavi M, Parmar P, Krishnamurthi R, Chugh S, et al. Global burden of stroke and risk factors in 188 countries, during 1990-2013: funding for the study. RS contributed substantial to the study conception and design, coordinated, planned and performed the intervention, including the patient assessments, processed data, and performed statistical work. The statistical analysis was performed in collaboration with TK and ER who also provided key content and critically reviewed the manuscript. JF and NP contributed to the study design, supervised data acquisition, and provided key content to the manuscript. HI and TC contributed to the data acquisition and provided key content to the discussion section and critically reviewed the manuscript. $\mathrm{SR}$ and KL contributed to the data analysis, interpretation of results, and critically reviewed the manuscript. The manuscript was drafted by RS, AV, and CK with contributions from all other authors. All authors gave final approval for the paper to be published.

\section{FUNDING}

This work was supported by The Associations of Danish Physiotherapists; Lions Clubs International Foundation, Denmark; Toyota-Fonden Denmark; The Foundation of Axel Muusfeldt; The Foundation of Aase \& Ejnar Danielsen; The A. P. Møller and Chastine Mc-Kinney Møller Foundation; and The Memorial Foundation of C. C Klestrup \& Wife Henriette Klestrup. The stationary bicycle used for exercise testing was kindly provided by ProTerapi A/S, Denmark. The funding parties did not have any influence on study design, data collection, analyses, or interpretation of the study. Novo Nordic Foundation grant number NNF18OC0031840.

\section{ACKNOWLEDGMENTS}

We thank the physiotherapists Signe Wildenskov, Tommy Olsen, Sus Ven, David Jonsson, Mette Mou Nielsen, and Gitte Sone Larsen for carrying out the cardiorespiratory fitness tests and nurse Agnete Hornnes for engaging in the study design. Additionally, the authors thank Jørgen Skotte for assisting with the use of accelerometers and both Ulla KjærulffHansen and Ulla Damgaard Munk for assisting with blood sample analyses.

\section{SUPPLEMENTARY MATERIAL}

The Supplementary Material for this article can be found online at: https://www.frontiersin.org/articles/10.3389/fneur. 2019.00664/full\#supplementary-material et al. One-year risk of stroke after transient ischemic attack or minor stroke. N Engl J Med. (2016) 374:1533-42. doi: 10.1056/NEJMoa1412981

4. Johnston SC, Amarenco P, Albers GW, Denison H, Easton JD, Evans SR, et al. Ticagrelor versus aspirin in acute stroke or transient ischemic attack. N Engl J Med. (2016) 375:35-43. doi: 10.1056/NEJMoa1603060 
5. O’Donnell MJ, Chin SL, Rangarajan S, Xavier D, Liu L, Zhang H, et al. Global and regional effects of potentially modifiable risk factors associated with acute stroke in 32 countries (INTERSTROKE): a case-control study. Lancet. (2016) 388:761-75. doi: 10.1016/S0140-6736(16)30506-2

6. Lee CD, Folsom AR, Blair SN. Physical activity and stroke risk: a meta-analysis. Stroke. (2003) 34:2475-81. doi: 10.1161/01.STR.0000091843.02517.9D

7. Wendel-Vos GC, Schuit AJ, Feskens EJ, Boshuizen HC, Verschuren WM, Saris $\mathrm{WH}$, et al. Physical activity and stroke. A meta-analysis of observational data. Int J Epidemiol. (2004) 33:787-98. doi: 10.1093/ije/dyh168

8. Adams RJ, Chimowitz MI, Alpert JS, Awad IA, Cerqueria MD, Fayad P, et al. Coronary risk evaluation in patients with transient ischemic attack and ischemic stroke: a scientific statement for healthcare professionals from the Stroke Council and the Council on Clinical Cardiology of the American Heart Association/American Stroke Association. Circulation. (2003) 108:1278-90. doi: 10.1161/01.CIR.0000090444.87006.CF

9. Sacco RL, Adams R, Albers G, Alberts MJ, Benavente O, Furie K, et al. Guidelines for prevention of stroke in patients with ischemic stroke or transient ischemic attack: a statement for healthcare professionals from the American Heart Association/American Stroke Association Council on Stroke: co-sponsored by the Council on Cardiovascular Radiology and Intervention: the American Academy of Neurology affirms the value of this guideline. Stroke. (2006) 37:577-617. doi: 10.1161/01.STR.0000199147.30016.74

10. Palmefors H, DuttaRoy S, Rundqvist B, Borjesson M. The effect of physical activity or exercise on key biomarkers in atherosclerosis-a systematic review. Atherosclerosis. (2014) 235:150-61. doi: 10.1016/j.atherosclerosis.2014.04.026

11. Warburton DE, Charlesworth S, Ivey A, Nettlefold L, Bredin SS. A systematic review of the evidence for Canada's Physical Activity Guidelines for Adults. Int J Behav Nutr Phys Act. (2010) 7:39. doi: 10.1186/1479-5868-7-39

12. Ellekjaer H, Holmen J, Ellekjaer E, Vatten L. Physical activity and stroke mortality in women. Ten-year follow-up of the Nord-Trondelag health survey, 1984-1986. Stroke. (2000) 31:14-8. doi: 10.1161/01.STR.31.1.14

13. Lindenstrom E, Boysen G, Nyboe J. Lifestyle factors and risk of cerebrovascular disease in women. The Copenhagen City Heart Study. Stroke. (1993) 24:1468-72. doi: 10.1161/01.STR.24.10.1468

14. Wannamethee G, Shaper AG. Physical activity and stroke in British middle aged men. BMJ. (1992) 304:597-601. doi: 10.1136/bmj.304.6827.597

15. Boysen G, Krarup LH. Benefits of physical activity for stroke survivors. Expert Rev Neurother. (2009) 9:147-9. doi: 10.1586/14737175.9.2.147

16. Bernhardt J, Dewey H, Thrift A, Donnan G. Inactive and alone: physical activity within the first 14 days of acute stroke unit care. Stroke. (2004) 35:1005-9. doi: 10.1161/01.STR.0000120727.40792.40

17. Mackay-Lyons MJ, Makrides L. Exercise capacity early after stroke. Arch Phys Med Rehabil. (2002) 83:1697-702. doi: 10.1053/apmr.2002.36395

18. Newitt R, Barnett F, Crowe M. Understanding factors that influence participation in physical activity among people with a neuromusculoskeletal condition: a review of qualitative studies. Disabil Rehabil. (2016) 38:1-10. doi: 10.3109/09638288.2014.996676

19. Nicholson S, Sniehotta FF, van Wijck F, Greig CA, Johnston M, McMurdo ME, et al. A systematic review of perceived barriers and motivators to physical activity after stroke. Int J Stroke. (2013) 8:357-64. doi: 10.1111/j.1747-4949.2012.00880.x

20. Langhorne $\mathrm{P}, \mathrm{Wu} \mathrm{O}$, Rodgers $\mathrm{H}$, Ashburn A, Bernhardt J. A Very Early Rehabilitation Trial after stroke (AVERT): a Phase III, multicentre, randomised controlled trial. Health Technol Assess. (2017) 21:1-120. doi: 10.3310/hta21540

21. Hannan AL, Hing W, Simas V, Climstein M, Coombes JS, Jayasinghe R, et al. High-intensity interval training versus moderate-intensity continuous training within cardiac rehabilitation: a systematic review and meta-analysis. Open Access J Sports Med. (2018) 9:1-17. doi: 10.2147/OAJSM.S150596

22. Kolmos M, Krawcyk RS, Kruuse C. Effect of high-intensity training on endothelial function in patients with cardiovascular and cerebrovascular disease: a systematic review. SAGE Open Med. (2016) 4:2050312116682253. doi: $10.1177 / 2050312116682253$

23. Schulz KF, Altman DG, Moher D. CONSORT 2010 statement: updated guidelines for reporting parallel group randomised trials. Int J Surg. (2011) 9:672-7. doi: 10.1016/j.ijsu.2011.09.004
24. Adams HP Jr., Bendixen BH, Kappelle LJ, Biller J, Love BB, Gordon DL, et al. Classification of subtype of acute ischemic stroke. Definitions for use in a multicenter clinical trial. TOAST. Trial of Org 10172 in Acute Stroke Treatment. Stroke. (1993) 24:35-41. doi: 10.1161/01.STR.24.1.35

25. Rosenberg GA, Wallin A, Wardlaw JM, Markus HS, Montaner J, Wolfson L, et al. Consensus statement for diagnosis of subcortical small vessel disease. $J$ Cereb Blood Flow Metab. (2016) 36:6-25. doi: 10.1038/jcbfm.2015.172

26. Govan L, Langhorne P, Weir CJ. Categorizing stroke prognosis using different stroke scales. Stroke. (2009) 40:3396-9. doi: 10.1161/STROKEAHA.109.557645

27. Buchheit M, Laursen PB. High-intensity interval training, solutions to the programming puzzle. Part II: anaerobic energy, neuromuscular load and practical applications. Sports Med. (2013) 43:927-54. doi: 10.1007/s40279-013-0066-5

28. Borg GA. Psychophysical bases of perceived exertion. Med Sci Sports Exerc. (1982) 14:377-81. doi: 10.1249/00005768-198205000-00012

29. Klarlund-Pedersen B, Andersen LB. Physical Activity-Manual on Disease Prevention and Treatment. (2018) Copenhagen: Sundhedsstyrelsen.

30. Foster C, Porcari JP, Ault S, Doro K, Dubiel J, Engen M, et al. Exercise prescription when there is no exercise test: the talk test. Kinesiology. (2018) 50:11581. doi: 10.26582/k.50.1.8

31. Woltmann ML, Foster C, Porcari JP, Camic CL, Dodge C, Haible S, et al. Evidence that the talk test can be used to regulate exercise intensity. J Strength Cond Res. (2015) 29:1248-54. doi: 10.1519/JSC.0000000000000811

32. Steen Krawcyk R, Vinther A, Petersen NC, Faber J, Hansen RH, Rostrup E, et al. Home-based aerobic exercise in patients with lacunar stroke: design of the HITPALS randomized controlled trial. Contemp Clin Trials Commun. (2019) 14:100332. doi: 10.1016/j.conctc.2019.100332

33. Okura T, Tanaka K. A unique method for predicting cardiorespiratory fitness using rating of perceived exertion. J Physiol Anthropol Appl Human Sci. (2001) 20:255-61. doi: 10.2114/jpa.20.255

34. Steen Krawcyk R, Vinther A, Caesar Petersen N, Kruuse C. "Graded cycling test with talk test" is a reliable test to monitor cardiovascular fitness in patients with minor stroke. J Stroke Cerebrovasc Dis. (2017) 26:494-9. doi: 10.1016/j.jstrokecerebrovasdis.2016.12.004

35. Smets EM, Garssen B, Bonke B, De Haes JC. The Multidimensional Fatigue Inventory (MFI) psychometric qualities of an instrument to assess fatigue. $J$ Psychosom Res. (1995) 39:315-25. doi: 10.1016/0022-3999(94)00125-O

36. Bech P, Rasmussen NA, Olsen LR, Noerholm V, Abildgaard W. The sensitivity and specificity of the Major Depression Inventory, using the Present State Examination as the index of diagnostic validity. J Affect Disord. (2001) 66:15964. doi: 10.1016/S0165-0327(00)00309-8

37. Bech P. Clinical Psychometrics. 2nd ed. Oxford: Wiley-Blackwell (2012).

38. Bech P, Olsen LR, Kjoller M, Rasmussen NK. Measuring well-being rather than the absence of distress symptoms: a comparison of the SF-36 Mental Health subscale and the WHO-Five Well-Being Scale. Int J Methods Psychiatr Res. (2003) 12:85-91. doi: 10.1002/mpr.145

39. Awata S, Bech P, Yoshida S, Hirai M, Suzuki S, Yamashita M, et al. Reliability and validity of the Japanese version of the World Health Organization-Five Well-Being Index in the context of detecting depression in diabetic patients. Psychiatry Clin Neurosci. (2007) 61:112-9. doi: $10.1111 / j .1440-1819.2007 .01619 . x$

40. Bergmann N, Ballegaard S, Holmager P, Kristiansen J, Gyntelberg F, Andersen LJ, et al. Pressure pain sensitivity: a new method of stress measurement in patients with ischemic heart disease. Scand J Clin Lab Invest. (2013) 73:373-9. doi: 10.3109/00365513.2013.785588

41. Ballegaard S, Petersen PB, Gyntelberg F, Faber J. The association between pressure pain sensitivity, and answers to questionnaires estimating psychological stress level in the workplace. A feasibility study. Scand $J$ Clin Lab Invest. (2012) 72:459-66. doi: 10.3109/00365513.2012.695023

42. Ballegaard S, Karpatschof B, Trojaborg W, Hansen AM, Magnusson G, Petersen PB. A simple and objective marker for stress. Scand J Clin Lab Invest. (2009) 69:713-21. doi: 10.3109/00365510903042734

43. Nasreddine ZS, Phillips NA, Bedirian V, Charbonneau S, Whitehead V, Collin I, et al. The Montreal Cognitive Assessment, MoCA: a brief screening tool for mild cognitive impairment. J Am Geriatr Soc. (2005) 53:695-9. doi: 10.1111/j.1532-5415.2005.53221.x 
44. Wong A, Xiong YY, Kwan PW, Chan AY, Lam WW, Wang K, et al. The validity, reliability and clinical utility of the Hong Kong Montreal Cognitive Assessment (HK-MoCA) in patients with cerebral small vessel disease. Dement Geriatr Cogn Disord. (2009) 28:81-7. doi: 10.1159/000232589

45. Hansen AS, Butt JH, Holm-Yildiz S, Karlsson W, Kruuse C. Validation of repeated endothelial function measurements using EndoPAT in stroke. Front Neurol. (2017) 8:178. doi: 10.3389/fneur.2017.00178

46. Kernan WN, Ovbiagele B, Black HR, Bravata DM, Chimowitz MI, Ezekowitz $\mathrm{MD}$, et al. Guidelines for the prevention of stroke in patients with stroke and transient ischemic attack: a guideline for healthcare professionals from the American Heart Association/American Stroke Association. Stroke. (2014) 45:2160-236. doi: 10.1161/STR.0000000000000024

47. Seifert-Held T, Pekar T, Gattringer T, Simmet NE, Scharnagl H, Bocksrucker $\mathrm{C}$, et al. Plasma midregional pro-adrenomedullin improves prediction of functional outcome in ischemic stroke. PLoS ONE. (2013) 8:e68768. doi: 10.1371/journal.pone.0068768

48. Katan M, Fluri F, Schuetz P, Morgenthaler NG, Zweifel C, Bingisser $\mathrm{R}$, et al. Midregional pro-atrial natriuretic peptide and outcome in patients with acute ischemic stroke. J Am Coll Cardiol. (2010) 56:1045-53. doi: 10.1016/j.jacc.2010.02.071

49. Butt JH, Rostrup E, Hansen AS, Lambertsen KL, Kruuse C. Induction of migraine-like headache, but not aura, by cilostazol in patients with migraine with aura. Brain. (2018) 141:2943-51. doi: 10.1093/brain/awy228

50. Abbasi F, Okeke Q, Reaven GM. Evaluation of fasting plasma insulin concentration as an estimate of insulin action in nondiabetic individuals: comparison with the homeostasis model assessment of insulin resistance (HOMA-IR). Acta Diabetol. (2014) 51:193-7. doi: 10.1007/s00592-013-0461-2

51. Andersen LG, Groenvold M, Jorgensen T, Aadahl M. Construct validity of a revised Physical Activity Scale and testing by cognitive interviewing. Scand $J$ Public Health. (2010) 38:707-14. doi: 10.1177/1403494810380099

52. Ainsworth BE, Haskell WL, Whitt MC, Irwin ML, Swartz AM, Strath SJ, et al. Compendium of physical activities: an update of activity codes and MET intensities. Med Sci Sports Exerc. (2000) 32 (Suppl. 9):S498-504. doi: 10.1097/00005768-200009001-00009

53. Skotte J, Korshoj M, Kristiansen J, Hanisch C, Holtermann A. Detection of physical activity types using triaxial accelerometers. J Phys Act Health. (2014) 11:76-84. doi: 10.1123/jpah.2011-0347

54. Nielsen SG, Buus L, Hage T, Olsen H, Walsoe M, Vinther A. The graded cycling test combined with the talk test is reliable for patients with ischemic heart disease. J Cardiopulm Rehabil Prev. (2014) 34:276-80. doi: 10.1097/HCR.0000000000000067

55. Tolstrup JS, Jensen MK, Tjonneland A, Overvad K, Gronbaek M. Drinking pattern and mortality in middle-aged men and women. Addiction. (2004) 99:323-30. doi: 10.1111/j.1360-0443.2003.00667.x

56. Crozier J, Roig M, Eng JJ, MacKay-Lyons M, Fung J, Ploughman M, et al. High-intensity interval training after stroke: an opportunity to promote functional recovery, cardiovascular health, and neuroplasticity. Neurorehabil Neural Repair. (2018) 32:543-56. doi: 10.1177/1545968318766663

57. Boyne P, Dunning K, Carl D, Gerson M, Khoury J, Rockwell B, et al. Highintensity interval training and moderate-intensity continuous training in ambulatory chronic stroke: feasibility study. Phys Ther. (2016) 96:1533-44. doi: $10.2522 /$ ptj.20150277

58. Lau KW, Mak MK. Speed-dependent treadmill training is effective to improve gait and balance performance in patients with sub-acute stroke. J Rehabil Med. (2011) 43:709-13. doi: 10.2340/16501977-0838

59. Pohl M, Mehrholz J, Ritschel C, Ruckriem S. Speed-dependent treadmill training in ambulatory hemiparetic stroke patients: a randomized controlled trial. Stroke. (2002) 33:553-8. doi: 10.1161/hs0202.102365

60. Hussain SR, Macaluso A, Pearson SJ. High-intensity interval training versus moderate-intensity continuous training in the prevention/management of cardiovascular disease. Cardiol Rev. (2016) 24:273-81. doi: 10.1097/CRD.0000000000000124

61. WHO. Global Recommendations on Physical Activity for Health. Geneva: World Health Organization (2010).

62. van Wijk I, Kappelle LJ, van Gijn J, Koudstaal PJ, Franke CL, Vermeulen M, et al. Long-term survival and vascular event risk after transient ischaemic attack or minor ischaemic stroke: a cohort study. Lancet. (2005) 365:2098-104. doi: 10.1016/S0140-6736(05)66734-7
63. Deijle IA, Van Schaik SM, Van Wegen EE, Weinstein HC, Kwakkel G, Van den Berg-Vos RM. Lifestyle interventions to prevent cardiovascular events after stroke and transient ischemic attack: systematic review and meta-analysis. Stroke. (2017) 48:174-9. doi: 10.1161/STROKEAHA.116.013794

64. D'Isabella NT, Shkredova DA, Richardson JA, Tang A. Effects of exercise on cardiovascular risk factors following stroke or transient ischemic attack: a systematic review and meta-analysis. Clin Rehabil. (2017) 31:1561-72. doi: 10.1177/0269215517709051

65. Saunders DH, Sanderson M, Hayes S, Kilrane M, Greig CA, Brazzelli M, et al. Physical fitness training for stroke patients. Cochrane Database Syst Rev. (2016) 3:CD003316. doi: 10.1002/14651858.CD003316.pub6

66. Wu S, Kutlubaev MA, Chun HY, Cowey E, Pollock A, Macleod MR, et al. Interventions for post-stroke fatigue. Cochrane Database Syst Rev. (2009) 2009:CD007030. doi: 10.1002/14651858.cd007030

67. Wang C, Redgrave J, Shafizadeh M, Majid A, Kilner K, Ali AN. Aerobic exercise interventions reduce blood pressure in patients after stroke or transient ischaemic attack: a systematic review and meta-analysis. Br J Sports Med. (2018) 2018:098903. doi: 10.1136/bjsports-2017-098903

68. Schumacher A, Peersen K, Sommervoll L, Seljeflot I, Arnesen H, Otterstad JE. Physical performance is associated with markers of vascular inflammation in patients with coronary heart disease. Eur J Cardiovasc Prev Rehabil. (2006) 13:356-62. doi: 10.1097/00149831-200606000-00010

69. Arba F, Gianninil A, Piccardi P, Biagini S, Palumbo V, Giusti B, et al. Small vessel disease and biomarkers of endothelial dysfunction after ischaemic stroke. Eur Stroke J. (2018) 1:1-8. doi: 10.1177/239698731 8805905

70. Cesari F, Sofi F, Caporale R, Capalbo A, Marcucci R, Macchi C, et al. Relationship between exercise capacity, endothelial progenitor cells and cytochemokines in patients undergoing cardiac rehabilitation. Thromb Haemost. (2009) 101:521-6. doi: 10.1160/TH08-10-0644

71. Lee BC, Hsu HC, Tseng WY, Su MY, Chen SY, Wu YW, et al. Effect of cardiac rehabilitation on angiogenic cytokines in postinfarction patients. Heart. (2009) 95:1012-8. doi: 10.1136/hrt.2008.153510

72. Billebeau G, Vodovar N, Sadoune M, Launay JM, Beauvais F, Cohen-Solal A. Effects of a cardiac rehabilitation programme on plasma cardiac biomarkers in patients with chronic heart failure. Eur J Prev Cardiol. (2017) 24:1127-35. doi: $10.1177 / 2047487317705488$

73. Pearson MJ, King N, Smart NA. Effect of exercise therapy on established and emerging circulating biomarkers in patients with heart failure: a systematic review and meta-analysis. Open Heart. (2018) 5:e000819. doi: 10.1136/openhrt-2018-000819

74. Boysen G, Krarup LH, Zeng X, Oskedra A, Korv J, Andersen G, et al. ExStroke Pilot Trial of the effect of repeated instructions to improve physical activity after ischaemic stroke: a multinational randomised controlled clinical trial. BMJ. (2009) 339:b2810. doi: 10.1136/bmj.b2810

75. Gregg EW, Jakicic JM, Blackburn G, Bloomquist P, Bray GA, Clark JM, et al. Association of the magnitude of weight loss and changes in physical fitness with long-term cardiovascular disease outcomes in overweight or obese people with type 2 diabetes: a post-hoc analysis of the Look AHEAD randomised clinical trial. Lancet Diabetes Endocrinol. (2016) 4:913-21. doi: 10.1016/S2213-8587(16)30162-0

76. Giroud M, Delpont B, Daubail B, Blanc C, Durier J, Giroud M, et al. Temporal trends in sex differences with regard to stroke incidence: the dijon stroke registry (1987-2012). Stroke. (2017) 48:846-9. doi: 10.1161/STROKEAHA.116.015913

Conflict of Interest Statement: The authors declare that the research was conducted in the absence of any commercial or financial relationships that could be construed as a potential conflict of interest.

Copyright (C) 2019 Steen Krawcyk, Vinther, Petersen, Faber, Iversen, Christensen, Lambertsen, Rehman, Klausen, Rostrup and Kruuse. This is an open-access article distributed under the terms of the Creative Commons Attribution License (CC BY). The use, distribution or reproduction in other forums is permitted, provided the original author(s) and the copyright owner(s) are credited and that the original publication in this journal is cited, in accordance with accepted academic practice. No use, distribution or reproduction is permitted which does not comply with these terms. 